\title{
Dynamic Response of Non-linear Beam Structures in Deterministic and Chaos Perspective
}

\author{
Anwar Dolu $^{1 *}$, Amrinsyah Nasution ${ }^{2}$ \\ ${ }^{1}$ Department of Civil Engineering, Tadulako University, Palu-Indonesia \\ ${ }^{2}$ Department of Civil Engineering, Bandung Institute of Technology, Bandung-Indonesia
}

( Received: August 20, 2018, Revised: December 21, 2018, Accepted: August 7, 2019 )

\begin{abstract}
The behavior of large deformation beam structures can be modeled based on non-linear geometry due to geometric nonlinearity mid-plane stretching in the presence of axial forces, which is a form a nonlinear beam differential equation of Duffing equation type. Identification of dynamic systems from nonlinear beam differential equations for deterministic and chaotic responses based on time history, phase plane and Poincare mapping. Chaotic response based on time history is very sensitive to initial conditions, where small changes to initial terms leads to significant change in the system, which in this case are displacement $x(t)$ and velocity $x^{\prime}(t)$ as time increases $(t)$. Based on the phase plane, it shows irregular and non-stationary trajectories, this can also be seen in Poincare mapping which shows strange attractor and produces a fractal pattern. The solution to this Duffing type equation uses the Runge-Kutta numerical method with MAPLE software application.
\end{abstract}

Keywords: Large displacement, Duffing equation, deterministic, chaos, Runge-Kutta, time history, phase plane, Poincare mapping, fractal.

\section{INTRODUCTION}

In general, real conditions of structural systems are mostly non-linear to a certain extent, in special cases it is simplified into linear system. In the liner system, causes and effects are linearly related, whereas in nonlinear system the relation is not comparable. The general form of differential equation which states non-linear vibration system is as follows [20][24];

$$
\frac{d^{2} x}{d t^{2}}+f\left(\frac{d x}{d t}, x, t\right)=0
$$

Dynamic analysis of non-linear structure systems in time history, can be deterministic, chaotic, and stochastic. Chaos response might be a special case of deterministic or stochastic response. In deterministic response, the behavior of the system in a long space of time can be well predicted in a closed form. In chaotic response, the behavior is very sensitive to the system initial conditions, where two conditions with insignificant difference of

\footnotetext{
* Corresponding author.

E-mail address: anwardolu1972@gmail.com
}

initial conditions will evolve into two far different circumstances.

In this study, the behavior of non-linear beam that received tension axial force $(N)$ on pin-roller support is observed. Numerical simulation uses variations in tension axial load, and initial conditions of support displacement $\mathrm{x}(0)$, with the aim to understand beam behavior based on displacement response and velocity in deterministic and chaotic perspective.

\section{NON-LINEAR BEAM DIFFERENTIAL EQUATION}

By reviewing a beam on pin-roller support experiencing initial axial force $N_{s}$ (tension or compression) according to Figure (1). The beam element experiences shear force $(Q)$, moment $(M)$, and total axial force $N . N=N_{s}+N_{d}$, with $N_{s}$ is the total of static axial force and $N_{d}$ is axial force due to vibration. Dynamic equilibrium equation is as follow [10];

$$
\frac{\partial M}{\partial x}+Q-\left(N_{s}+N_{d}\right) \frac{\partial v}{\partial x}=0
$$




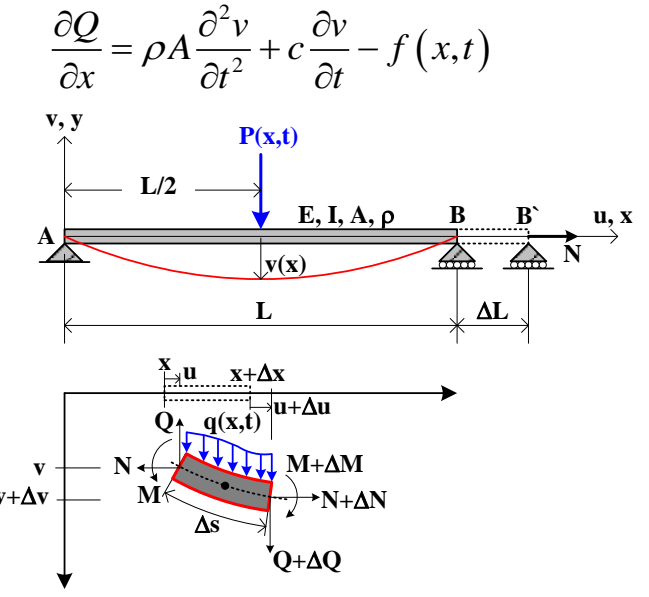

Figure 1. Vibrating beam equilibrium system with axial force

By solving equation (2) and (3), obtained;

$$
\rho A \frac{\partial^{2} v}{\partial t^{2}}+c \frac{\partial v}{\partial t}+E I \frac{\partial^{4} v}{\partial x^{4}}-N \frac{\partial^{2} v}{\partial x^{2}}=f(x, t)
$$

The first, second, and third terms on the left side of the equation is related to the linear behavior of the Euler-Bernoulli Beam Theory, while the fourth term is non-linear component due to axial force parallel to the beam. Axial force due to $N$,

$$
N=N_{s}+N_{d}
$$

Axial force $N_{d}$ [21],

$$
N_{d}=\frac{E A}{2 L} \int_{0}^{L}\left(\frac{\partial v}{\partial x}\right)^{2} d x
$$

Therefore, axial force due to $\mathrm{N}$ according to equation (5) and equation (6) can be written as;

$$
N=N_{s}+\frac{E A}{2 L} \int_{0}^{L}\left(\frac{\partial v}{\partial x}\right)^{2} d x
$$

By substituting equation (7) to equation (4), the non-linier beam differential equation with axial force $N s$ which can be compression axial force $(+N)$ or tension axial force $(-N)$.

$$
\begin{aligned}
& \rho A \frac{\partial^{2} v}{\partial t^{2}}+c \frac{\partial v}{\partial t}+E I \frac{\partial^{4} v}{\partial x^{4}} \\
& -\left[N_{s}+\frac{E A}{2 L} \int_{0}^{L}\left(\frac{\partial v}{\partial x}\right)^{2} d x\right] \frac{\partial^{2} v}{\partial x^{2}}=P(x, t)
\end{aligned}
$$

Equation (8) might be solved in a form of eigenfunction [6][7][17]:

$$
v(x, t)=\sum_{n=1}^{\infty} \Phi_{n}(x) q_{n}(t)
$$

By Galerkin process [19][28];

$$
\int_{D} R(x) W_{n}(x) d x=0 \quad n=1,2,3, \ldots
$$

Here $D$ states the domain of structures being reviewed. $W n$ is weighting function, $R n$ is residue. In Galerkin method, experiment function is the same as weighting function, hence;

$$
W_{n}=\Phi_{n} \quad n=1,2,3, \ldots
$$

According to equation (10) and equation (11) then obtained:

$$
\int_{0}^{L} R(x) \Phi_{n}(x) d x=0 \quad n=1,2,3, \ldots
$$

Solution of equation (8) according to the equation $(9,10,11,12)$ for the first mode review $(\mathrm{n}=1)$

$$
\begin{aligned}
& \rho A \frac{d^{2}}{d t^{2}} q_{1}(t) \Phi_{1}(x)+c \frac{d}{d t} q_{1}(t) \Phi_{1}(x) \\
& +E I q_{1}(t) \frac{d^{4}}{d t^{4}} \Phi_{1}(x) \\
& +q_{1}(t) \frac{d^{2}}{d x^{2}} \Phi_{1}(x)\left[T s+\frac{E A}{2 L} \int_{0}^{L} q_{1}(t)^{2}\left(\frac{d}{d x} \Phi_{1}(x)\right)^{2} d x\right] \\
& -F \cos (\Omega t)=0
\end{aligned}
$$

With limit conditions according to Figure 1 :

$$
\Phi(x=0, L)=0 \quad ; \quad \Phi^{\prime \prime}(x=0, L)=0
$$

Hence, obtained second-order differential equation for mode 1 ;

$$
\begin{aligned}
& \frac{1}{2} \rho A L \ddot{q}(t)+\frac{1}{2} c L \dot{q}(t)+\frac{\pi^{4} E I}{2 L^{3}}\left\{1+\frac{T_{s}}{E I \pi^{2}}\right\} q(t) \\
& +\frac{E A \pi^{4}}{8 L^{3}} q^{3}(t)=F \cos (\Omega t)
\end{aligned}
$$

With

$$
\begin{aligned}
& M=\frac{\rho A L}{2} ; C=\frac{c L}{2} ; \quad k_{1}=\frac{E I \pi^{4}}{2 L^{3}} \\
& k_{1 T}=\left[1 \pm T_{s}\left(\frac{L^{2}}{E I \pi^{2}}\right)\right] k_{1} \quad ; \quad k_{3}=\frac{E A \pi^{4}}{8 L^{3}}
\end{aligned}
$$

On equation (15) $\mathrm{T}_{\mathrm{s}}$ will be positive if the axial force is compression force and will be negative if the axial force is tension force. Therefore,

$$
m \ddot{q}(t)+c \dot{q}(t) \pm k_{1 T} q(t)+k_{3} q^{3}(t)=F \cos (\Omega t)
$$

Or written as,

$$
\ddot{q}(t)+2 \zeta \omega_{n} \dot{q}(t) \pm \omega_{n}^{2} q(t)+\beta q^{3}(t)=\bar{F} \cos (\Omega t)
$$

\section{Solution of Vibration Differential Equations}

The solution of linear differential equations generally uses analytical methods (exact methods and approaches) and numerical methods, but nonlinear differential equations will be more easily solved using approach method and numerical method, in many cases numerical method is widely used especially the Runge-Kutta method. In RungeKutta method [24], second-order differential equations are first reduced to two first-order equations. According to equation (18), which can be written as follow;

$$
\dot{q}=r \quad ; \quad \ddot{q}=\bar{F} \cos (\Omega t)-2 \zeta \omega_{n} \dot{q}-\omega_{n}^{2} q-\beta q^{3}
$$

Taking $\dot{q}=r$, the equation is reduced to two firstorder equations;

$$
\dot{q}=r \quad ; \quad \dot{r}=f(q, r, t)
$$


For the application of Runge-Kutta numerical method, MATLAB software can be used with the code of ode45 and MAPLE with rkf45.

\section{DINAMYC SYSTEM IDENTIFICATION}

\section{Deterministic Response}

Deterministic response is a process where momentary values and long-term characteristic can be determined or predicted in the domain of space and time. Deterministic response can be identified using the quantitative and qualitative method [1][2][13][14][15][23]. Quantitative identification uses 1). Frequency response, 2). Time history response, and qualitative identification uses (1). Phase plane history, (2). Poincare Mapping (Poincare map), and (3) Fourier Spectrum. Time history response is the solution of non-linear and linear differential equations obtained from analytical and numerical methods. Phase plane is used to identify the dynamic system behavior, where the motion equation of the dynamic system does explicitly contain time called the autonomous system. Figure (2) shows the trajectory of linear vibration equations, which is a certain ellipse for a total energy $\mathrm{E}$ from kinetic and potential energy of the system:

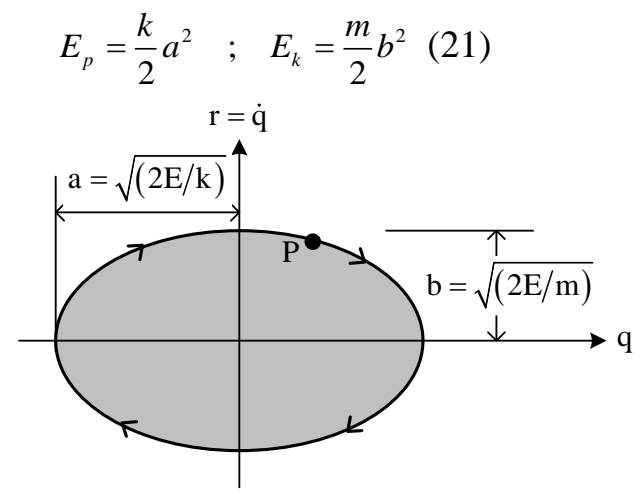

Figure 2. Phase Diagram

According to equation (18), the phase plane can be written in two first-order equation. As the Poincare mapping uses;

$$
\begin{aligned}
& \dot{q}=r \quad ; \quad \dot{r}=\ddot{q}=-\omega_{n}^{2} q-\beta q^{3}+\bar{F} \cos (\Omega t) \\
& \dot{t}=1
\end{aligned}
$$

Started at $\mathrm{t}=\mathrm{t}_{0}$, points are plotted on a space phase surface with the interval period of $\mathrm{T}$

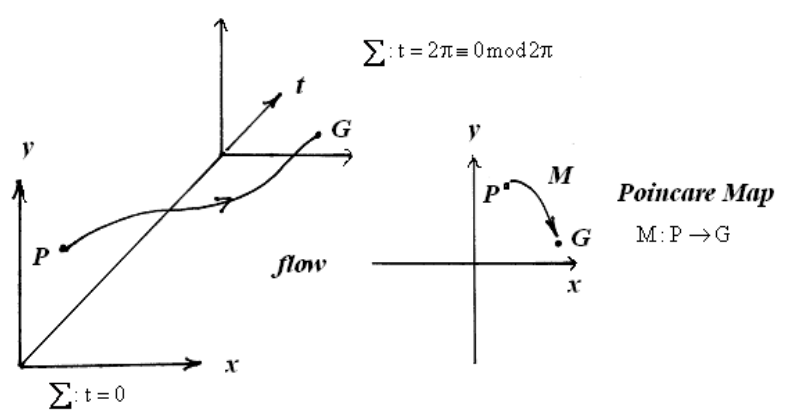

Figure 3. Poincare Mapping

\section{Power Spectrum (Fourier Spectrum)}

Another important identification method is power spectrum. Suppose a differential equation that has a solution of $q(t)$ is dependent to time, which applies to all $\mathrm{q}(-\infty<1<\infty)$. Fourier transformation $\mathrm{Q}$ (f) from $q(t)$ is used to analyze the frequency,

$$
Q(f)=\int_{-\infty}^{\infty} q(t) e^{-2 \pi i t} d t
$$

Where $f$ is frequency in hertz (second/cycle). Given $\mathrm{Q}(f)$ for all $f$, hence $\mathrm{q}(\mathrm{t})$ can be solved using Fourier invers transformation

$$
q(t)=\int_{-\infty}^{\infty} Q(f) e^{2 \pi i f t} d f
$$

Using Fourier approach;

$$
Q\left(f_{k}\right)=\sum_{n=0}^{N-1} q_{n} e^{-2 \pi i i_{k} t_{n}} T_{s}=T_{s} \sum_{n=0}^{N-1} q_{n} e^{-2 \pi i k n / N}
$$

Hence power spectrum can be defined as;

$$
S_{N}(k)=\frac{1}{N}\left|Q_{k}\right|^{2}
$$

\section{Chaotic response}

The term "Chaos" was first popularized by Tien Yien Li and James Yorke in 1975. The term Chaos is defined as follows: 1). Sort of regularity without periodicity. 2). Random repetitive behavior in deterministic system 3). Simple model capabilities, which do not contain random elements, to produce highly irregular behavior. Chaos is a dynamic phenomenon. This phenomenon was first studied by Poincare (1854-1912). A well-known example of chaos problems is the weather behavior from Lorenz [12] with butterfly effect, where the consequence of the discovery is that "two conditions where the amount of difference is not significant at the beginning will evolve into two major differences in the future".

In many cases, non-linear response is a chaotic symptom. Nowadays, this symptom is widely studied. Ueda describes chaotic phenomena in dynamic systems governed by Duffing equation in the late 1970s, which addresses the non-linear dynamic behavior related to chaotic symptoms [2] Moon, Nayfeh, Awrejcewicz discussed Duffing 
model on beam with the consequence of chaotic response [3][15][16].

The steps in qualitative and quantitative deterministic response might be followed to identify the system response of chaotic motions.

\section{CASE STUDY}

By reviewing continuous beam model that accepts axial loads according to equation (16-18), second order differential equation is obtained with third order nonlinear stiffness. Applying axial load variations during critical load condition on continuous beam model, Euler (Pcr):

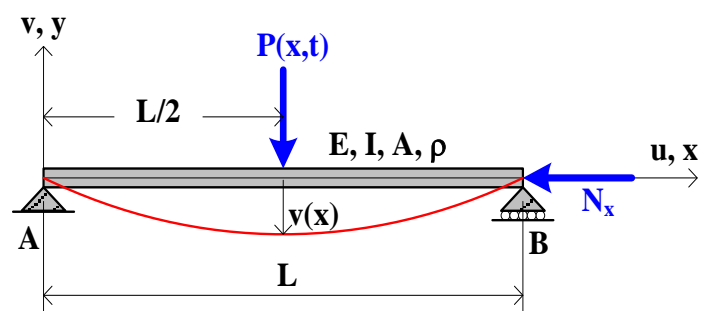

Figure 4. Pin-roller support geometry with axial loads $(N x)$

As a sensitivity test, the initial conditions of displacement and speed is set to be; $\mathrm{x}(0)=$ and $\mathrm{v}(0)$ $=0$, then it becomes; $\mathrm{x}(0)=0.01$ and $\mathrm{v}(0)=0$

$$
\begin{aligned}
& \frac{d^{2}}{d t^{2}} x(t)+0.04436 \frac{d}{d t} x(t)+0.4015 x(t)^{3} \\
& =9.7941 \cos (0.50 t)
\end{aligned}
$$

Runge-Kuta method, with Maple software (code rkf45), is used to solved equation (27). For initial conditions simulation type (1), $\mathrm{x}(0)=0$ and type $(2), x(0)=0.01$. The solution of equation (27) according to figure (5a) shows that, at time $\mathrm{t}=0$ to $\mathrm{t}$ $=75 \mathrm{~s}$, the effect of initial conditions is not significant for both initial conditions $\mathrm{x}(0)$ (types 1 and 2), but as time increases, the condition of the system will be very different. By reviewing the phase space on Figure 5b, it shows that the nonstationary pattern with time is elongated. According to historical time response (Figure 5a), the image phase space $(5 \mathrm{~b})$ in the non-linear model (equation 27) shows sensitive dependency to initial conditions.

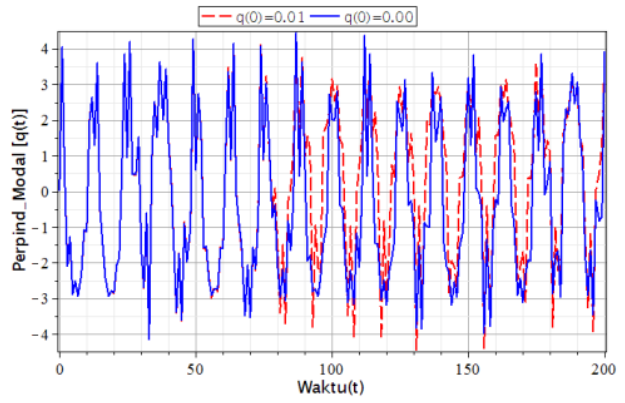

Figure 5a. Time history displacement

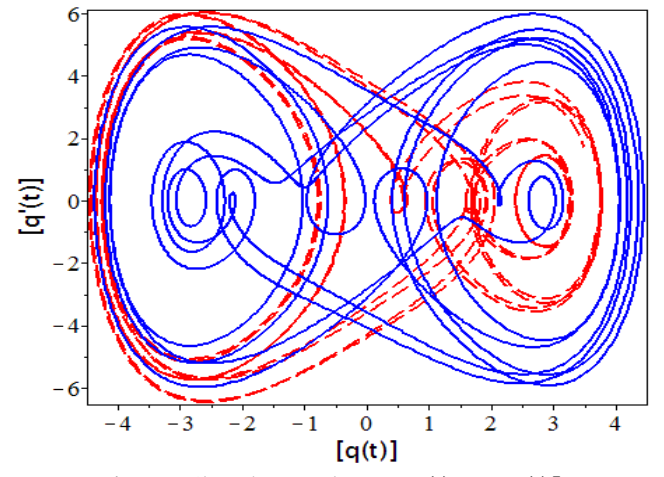

Figure 5b. Phase Plane [ q(t) vs q'(t)]

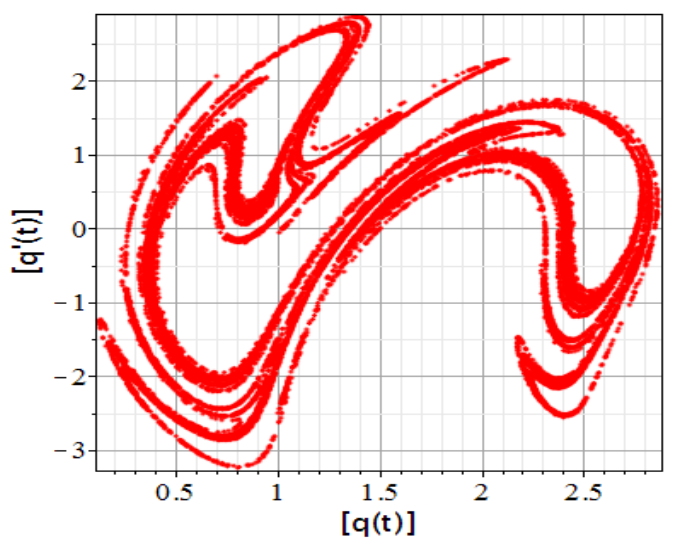

Figure 6a. Poincare Mapping

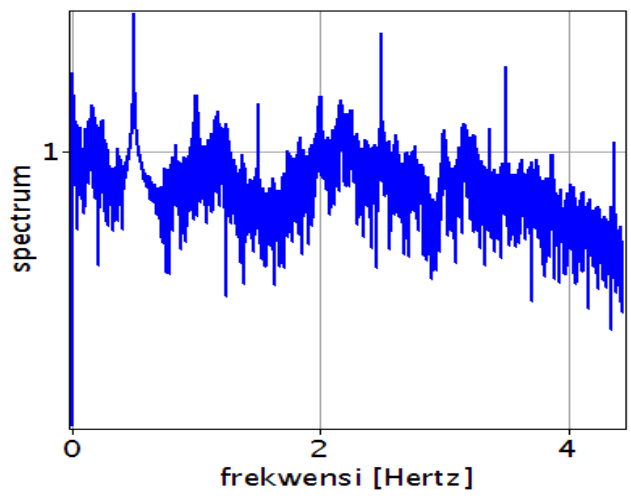

Figure 6b. Fourier Spectrum

That sensitive dependency, where only small changes to initial conditions, in fact significantly affects the system response; displacement $\mathrm{x}(\mathrm{t})$ and speed $v(t)$ as time flows $(\mathrm{t})$. This behavior is called chaotic symptoms. The chaotic symptoms can also be seen in the Fourier spectrum (6b) and Poincare mapping (6a) which shows strange attractors and fractal patterns with 50,000 points mapping.

\section{Bifurcation diagram}

With bifurcation diagram simulation, for critical load increasement of Per $=0.17$, Euler critical load according to equation (16), Per $=610.69 \mathrm{~kg}$. It can be seen that chaotic conditions occur when the load $\mathrm{P}=\mathrm{Pcr}$, and $\mathrm{P}>\mathrm{Pcr}$ (see figure 7). The system returns to be deterministic after the load $P>800 \mathrm{~kg}$. 


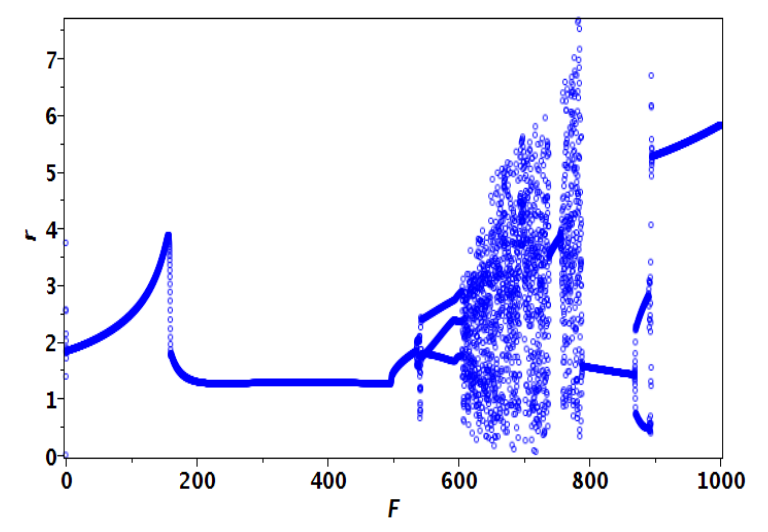

Figure 7. Bifurcation diagram with variations in axial load

\section{Deterministic and chaotic response simulations}

As comparison of deterministic and chaotic responses, with simulation of variations in external loads according to the following equation (16-18)

$$
\begin{aligned}
& \frac{d^{2}}{d t^{2}} x(t)+0.04436 \frac{d}{d t} x(t)-0.3222 x(t) \\
& +0.4015 x(t)^{3}=3.2647 \cos (0.50 t) \\
& \frac{d^{2}}{d t^{2}} x(t)+0.04436 \frac{d}{d t} x(t)-0.3222 x(t) \\
& +0.4015 x(t)^{3}=9.79415 \cos (0.50 t)
\end{aligned}
$$

According to case 1 in equation (28), the change in initial condition, which is the displacement $x(t)$ does not affect the response of the system even with a long period of time (Figure 8a). In case number 2 of equation (29), with a slight change in the initial condition (displacement $\mathrm{x}(\mathrm{t})$ ) ranging from $\mathrm{x}(0)=$ 0.00 to $\mathrm{x}(0)=0.01$, the response of the two systems will vary over a long period of time (see Figure $8 b$ ).

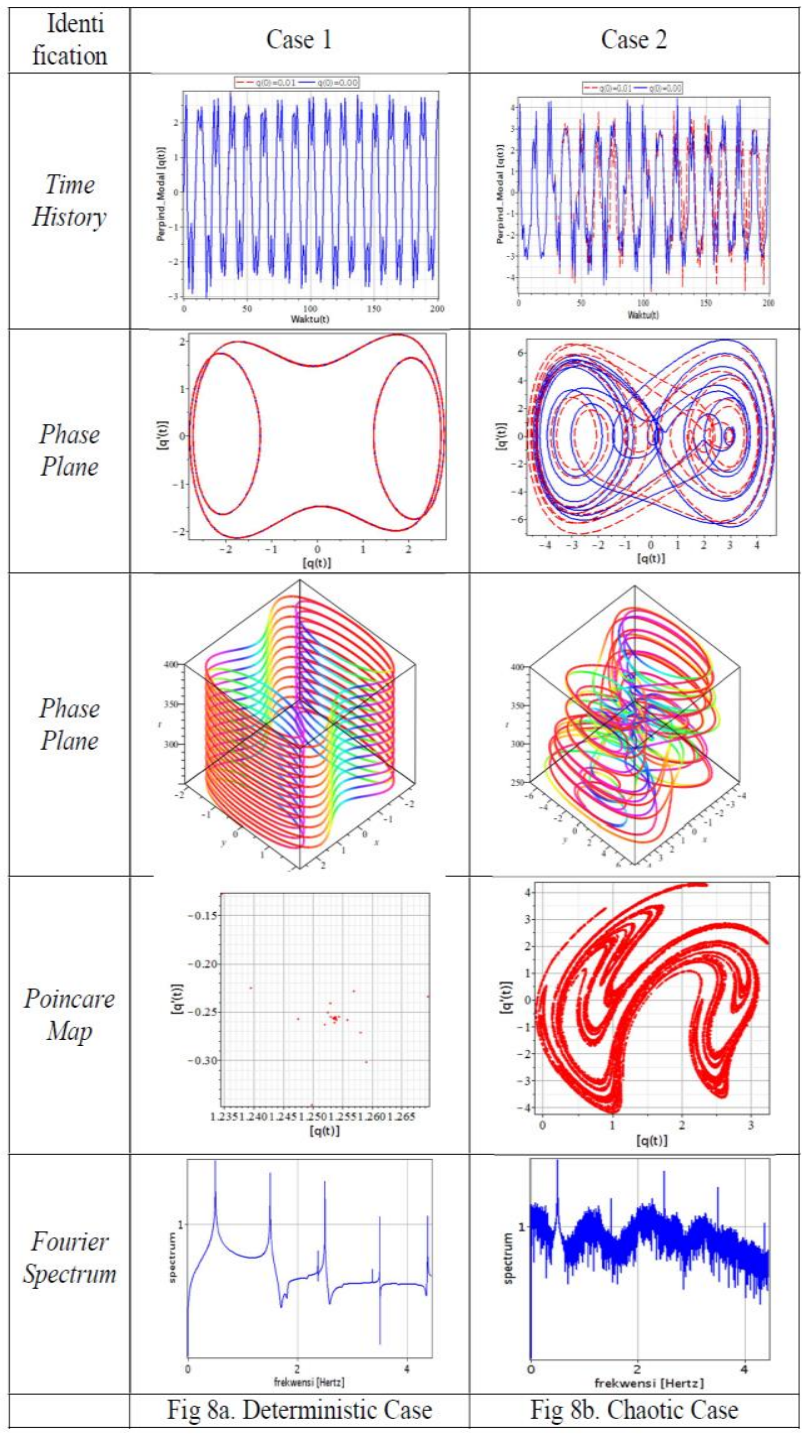

\section{CONCLUSION}

Beams that receive axial loads, the stability problem is very dominant, in the case of static Euler bending (Pcr). In dynamic case, system response is the system parameter function, where system response can either be deterministic or chaotic response. In deterministic response, small changes in initial conditions have no effect on the response of the system as time increases. On the other hand, small changes in initial conditions of chaotic response will greatly affect the response of the system as time increases. Changes in initial conditions on beams are closely related to the application of construction that is method of construction, in this case is the displacement or shift in joints, or environmental influences such as temperature, wind load, earthquake and other disturbances. 


\section{ACKNOWLEDGEMENT}

Author would like to express sincere gratitude to the team of promoter lecturers and the lecturer reviewer dissertation team, as well as various parties who contribute to the writing of this paper

\section{REFERENCES}

[1] Argyris, J., Faust, G., Haase, M. (1994). An Exploration of Chaos, An Introduction for Natural Scientists and Engineers. NorthHolland

[2] Asayama, S., Aizawa, M. (2000). Respons of Base Isolated Structure in Chaotic Dynamic System Under Earthquake Motion with Large Amplitude.12th World Conference on Earthquake Engineering, Auckland, New Zealand

[3] Awrejcewicz, J., Krysko, V.A. (2008). Chaos in Structural Mechanics, Berlin Heidelberg, Springer-Verlag

[4] Bayat, M., Pakar, I., Shahidi, M. (2011). Analysis of Nonlinear Vibration of Coupled Systems with Cubic Nonliearity. Journal Mechanica.17(6):620-629.

[5] Belendez, A., Bernabeu, G., Frances, J., Mendez, DI., Marini, S. (2010). An Accurate Closed-form Approximate Solution for the Quintic Duffing Oscillator Equation. Journal Mathematical and Computing Modelling, Vol. 52, Issues 3-4, August 2010 : pp. 637 - 641.

[6] Clough, R.W., Penzien, J. (2003). Dynamics of Structure. Third Edition. Computers \& Structures, Inc. University Berkeley. USA.

[7] Chopra, A.K., (2001). Dynamics Structure ; Theory and Application to Earthquake Engineering, Second Edition, byPrentice Hall.

[8] Ens, R.H., McGuire, G.C. (2001). Nonlinear Phisics with Mathematica for Scientiest and Engineers. Birkhauser, Boston. USA.

[9] Fahzi, A., Belhaq, M., Lakrad, F. (2009). Suppression of Hysteresis in a Forced Van der Pol - Duffing Oscillator. Journal Commun Nonlinear Sci Numer Simulat 14 (2009) : 1609 $-1616$.

[10] Kovacic, I,. Brennan, MJ. (2011). The Duffing Equation - Nonlinear Oscillator and their Behaviour. London, John Wiley \& Sons.

[11] Lai, S.K., Lim, C.W., Wu, B.S., Wang, C., Zeng, Q.C., He, X.F. (2009). NewtonHarmonic Balancing Approach for Accurate Solutions to Nonlinear Cubic-Quintic Duffing Oscillators. Applied Mathematical Modelling 33, 852-866.
[12] Lorenz, E.N. (1963). Deterministic Nonperiodic Flow. Journal of the Atmospheric Sciences, Volume 20. March 1963. pp 130 -141

[13] Lynch, S. (2010). Dynamical Systems with Applications using Maple, second edition. Birkhauser Boston - Springer Science Bussines Media. USA.

[14] Moon, F.C. (1983). Chaotic Vibrations of a Beam with Non-LinearBoundary Conditions. Int Journal Non-Linear Mechanics, Vol. 18, No. 6, pp. 465-477. Great Britain.

[15] Moon, F.C. (2004). Chaotic and Fractal Dynamics, An Introduction for Applied Scientist and Engineers.Wiley-VCH Verlag GmbH\&Co.KgaA. Weinheim.

[16] Nayfeh, A.H., Pai, P.F. (2004). Linear dan Nonlinear Structural Mechanics. John Wiley \& Sons. USA.

[17] Paz, M. (1996). Dinamika Struktur ; Teori dan Perhitungan. Penerbit Erlangga. Jakarta.

[18] Pai, P.F. (2006). Highly Flexible Structures ; Modeling, Computation, and Experimentation. AIAA Inc, Reston, Virginia

[19] Portela, A., Charafi, A. (2002). Finite Elements using MAPLE, A Symbolic Programming Approach. Springer-Verlag, Berlin. Germany

[20] Rand, Richard H. (2012). Lecture Notes on Nonlinear Vibrations. https://ecommons.cornell.edu/bitstream/handle/ 1813/28989/NonlinearVibrations_ver53.pdf

[21] Sathyamoorthy, M. (1998). Nonlinear Analysis of Structures. CRC Press LLC. USA

[22] Starosvetsky, Y., Gendelman, O.V. (2010). Bifurcations of Attractors in Forced System with Nonlinear Energy Sink: the Effect of Mass Asymmetry. Journal Nonlinear Dynamic 59 : 711-731.

[23] Thompson, J.M.T. and Stewart, H.B. (1986), Nonlinear Dynamics and Chaos Geometrical Methods for Engineers and Scientists. John Wiley \& Sons.New York

[24] Thompson, W.T. (1982). Theory of Vibration with Applications. Second Edition. Prentice Hall of India.

[25] Ueda, Y. (1991). The Road to Chaos. Aerial Press, Inc. Santa Cruz.

[26] Urabe, M. (1969). Numerical Investigation of Subharmonic Solutions to Duffing's Equation. Publ. RIMS, Kyoto Univ. Vol. 5, pp. 79-112.

[27] Zardar, Ziauddin,. Abrams, Iwona, (1998), Chaos for Beginners, Cambridge, Inggris, Icon Brooks

[28] Zienkiewicz, O.C., Morgan, K. (1983). Finite Element and Approximation. John Wiley \& Sons 